\title{
FREQUENCY-DOMAIN SUBSPACE IDENTIFICATION OF NONLINEAR MECHANICAL SYSTEMS
}

\author{
Jean-Philippe Noël ${ }^{1}$, Gaëtan Kerschen ${ }^{2}$
}

\begin{abstract}
The objective of the present paper is to address the identification of a real-life strongly nonlinear space structure, the EADS-Astrium SmallSat spacecraft. To this end, a new nonlinear subspace identification method formulated in the frequency domain is exploited, referred to as the FNSI method. The frequency response functions of the underlying linear spacecraft and the amplitudes of the nonlinear internal forces are estimated based on a periodic-random data set corrupted by noise. This application is challenging for several reasons, including high modal density, highly non-proportional damping and the non-smooth nature of the nonlinearities.
\end{abstract}

Keywords: Nonlinear system identification, subspace method, frequency domain, space structure.

\section{INTRODUCTION}

The identification of linear and time-invariant systems is a discipline that has evolved considerably during the last forty years. The techniques available today, e.g. [1] and [2], are really quite sophisticated and advanced. In particular, subspace algorithms are powerful identification methods that are naturally applicable to multi-input, multi-output systems. They are routinely used for experimental and operational modal analysis [3], but also for advanced signal processing such as damage detection and structural health monitoring [4].

However, because engineering structures are known to be prone to nonlinearity, there is a crucial need for extending linear subspace methods to a practical nonlinear analogue. In this context, the first contribution is due to Lacy and Bernstein [5], who derived a time-domain algorithm applicable to mechanical systems, later improved by Marchesiello and Garibaldi [6]. More recently, a dual approach has been developed in the frequency domain, referred to as the frequency-domain nonlinear subspace identification (FNSI) method [7], whose main advantage is the possibility to discriminate frequency samples according to information content and signal-to-noise ratio, thereby increasing the accuracy and reducing the computational burden.

The objective of the present paper is to address the identification of a real-life nonlinear space structure, the SmallSat spacecraft developed by EADS-Astrium, using the FNSI method. The frequency response functions of the underlying linear spacecraft and the amplitudes of the nonlinear internal forces will be estimated by this approach based on a periodic-random data set corrupted by noise. The SmallSat spacecraft comprises a strongly nonlinear component consisting of eight mechanical stops limiting the motion of an inertia wheel mounted on an elastomeric interface. This application is challenging for several reasons, including high modal density, highly non-proportional damping and the non-smooth nature of the nonlinearities. The estimation of the clearances beyond which the mechanical stops are reached will also be found to be a distinct challenge, tackled through the use of a goodness-of-fit indicator capable of detecting modelling errors.

\footnotetext{
${ }^{1}$ Space Structures and Systems Lab, University of Liège, Belgium, jp.noel@ulg.ac.be

${ }^{2}$ Space Structures and Systems Lab, University of Liège, Belgium, g.kerschen@ulg.ac.be
} 


\section{SUBSPACE IDENTIFICATION OF NONLINEAR MECHANICAL SYSTEMS IN THE FREQUENCY DOMAIN}

\subsection{State-space model and problem statement}

As described in the review paper [8], the identification of nonlinear mechanical systems can be seen as a three-step problem encapsulating nonlinearity detection, characterisation and parameter estimation. Following the detection step whose goal is obvious, characterisation is concerned with nonlinearity localisation and model selection. The model parameters are then estimated through least-squares fitting, or nonlinear optimisation. The aim of the FNSI method is to address this latter step and so to estimate nonlinear stiffness and damping coefficients together with the frequency response function (FRF) matrix of the underlying linear system.

The vibrations of nonlinear systems are governed by the time-continuous model

$$
M \ddot{q}(t)+C \dot{q}(t)+K q(t)+f(q(t), \dot{q}(t))=p(t)
$$

where $M, C, K \in \mathbb{R}^{r \times r}$ are the linear mass, damping and stiffness matrices, respectively; $q(t)$ and $p(t) \in \mathbb{R}^{r}$ are the generalised displacement and force vectors, respectively; $f(t) \in \mathbb{R}^{r}$ is the nonlinear restoring force vector, and $r$ is the number of degrees of freedom (DOFs) of the structure obtained after spatial discretisation. The amplitude, direction, location and frequency content of the excitation $p(t)$ determine in which regime the structure behaves. As in reference [9], the joint effect of the $s$ lumped nonlinearities in the system is modelled using a summation of the form

$f(q(t), \dot{q}(t))=\sum_{j=1}^{s} \mu_{j} b_{j} g_{j}(q(t), \dot{q}(t))$

Each term contains an unknown nonlinear coefficient $\mu_{j}$ and the corresponding functional form $g_{j}(t)$, which is assumed to be known. Nonlinearity localisation is specified using a vector of Boolean values, $b_{j} \in \mathbb{R}^{r}$. In the technical literature about subspace methods, first-order state-space models are preferred to the second-order description of the dynamics in Equation (1), because of the intrinsic capability of a state-space model to encompass multi-input, multi-output systems. Considering that displacements are measured and defining the state vector $x=\left(q^{T} \dot{q}^{T}\right)^{T} \in \mathbb{R}^{n}$, the equations of motion are recast into

$$
\left\{\begin{array}{c}
\dot{x}(t)=A_{c} x(t)+B_{c}^{n l} g(t)+B_{c} p(t) \\
q(t)=C_{c} x(t)+D_{c} p(t)
\end{array}\right.
$$

where subscript $c$ stands for continuous-time; $A_{c} \in \mathbb{R}^{n \times n}, B_{c}^{n l} \in \mathbb{R}^{n \times s}, B_{c} \in \mathbb{R}^{n \times r}, C_{c} \in \mathbb{R}^{r \times n}$ and $D_{c} \in \mathbb{R}^{r \times r}$ are the state, nonlinear coefficient, input, output and direct feed-through matrices, respectively; $g(t) \in \mathbb{R}^{s}$ gathers the basis functions $g_{j}(t)$, and $n=2 r$. State-space and physical-space matrices correspond through the relations

$$
\begin{aligned}
& A_{c}=\left(\begin{array}{cc}
0^{r \times r} & I^{r \times r} \\
-M^{-1} K & -M^{-1} C
\end{array}\right) \quad B_{c}^{n l}=\left(\begin{array}{cccc}
0^{r \times 1} & 0^{r \times 1} & \ldots & 0^{r \times 1} \\
-\mu_{1} M^{-1} b_{1} & -\mu_{2} M^{-1} b_{2} & \ldots & -\mu_{s} M^{-1} b_{s}
\end{array}\right) \\
& B_{c}=\left(\begin{array}{c}
0^{r \times r} \\
-M^{-1}
\end{array}\right) \quad C_{c}=\left(\begin{array}{lll}
I^{r \times r} & 0^{r \times r}
\end{array}\right) \quad D_{c}=0^{r \times r}
\end{aligned}
$$

where 0 and $I$ are zero and identity matrices, respectively.

Given measurements of $p(t)$ and $q(t)$, the FNSI method first delivers an appropriate model order $n$ and the five system matrices $A_{c}, B_{c}^{n l}, B_{c}, C_{c}$ and $D_{c}$. The estimation of the nonlinear coefficients $\mu_{j}$ 
and of the FRF matrix is subsequently achieved using the conversion scheme from state space to physical space outlined in Section 2.5.

\subsection{Equivalent linear identification through feedback}

The FNSI method relies on the feedback interpretation of the dynamics governed by Equation (1) proposed in [9]. It consists in moving the nonlinear forces to the right-hand side of this equation and viewing them as external forces applied to the underlying linear structure. Thus, the internal forces that are nonlinear functions of the outputs act as a feedback to the linear open-loop system. Considering Equations (3), this interpretation boils down into the concatenation of $g(t)$ and $p(t)$ into a single extended input vector $e(t) \in \mathbb{R}^{s+r}$ :

$\left\{\begin{array}{c}\dot{x}(t)=A_{c} x(t)+B_{c}^{e} e(q(t), \dot{q}(t), p(t)) \\ q(t)=C_{c} x(t)+D_{c}^{e} e(t)\end{array}\right.$

where $B_{c}^{e}=\left(B_{c}^{n l} B_{c}\right) \in \mathbb{R}^{n \times(s+r)}$ and $D_{c}^{e}=\left(0^{r \times s} D_{c}\right) \in \mathbb{R}^{r \times(s+r)}$. The feedback formulation is particularly appealing because the inverse problem to be solved is now equivalent to the widelystudied linear state-space identification problem. However, the presence of nonlinearities in inputoutput (I-O) data requires the interpretation and use of the FNSI algorithm to be tailored, as discussed throughout this paper.

As extensively substantiated by Pintelon and Schoukens in [10], tackling system identification problems in the frequency domain is an attractive and versatile alternative. For improved numerical conditioning [11], a discrete-time translation of Equations (5) is first considered, before applying the discrete Fourier transform (DFT). Provided that the time signal $v(t)$ is periodic and observed over an integer number of periods in steady-state conditions, its DFT $V(k)$ is given by

$V(k)=\frac{1}{\sqrt{M}} \sum_{t=0}^{M-1} v(t) e^{-j 2 \pi k t / M}$

where $M$ is the number of recorded time samples, $k$ the frequency line and $j$ the imaginary unit. Equations (5) eventually write

$\left\{\begin{array}{c}z_{k} X(k)=A_{d} X(k)+B_{d}^{e} E(k) \\ Q(k)=C_{d} X(k)+D_{d}^{e} E(k)\end{array}\right.$

where subscript $d$ stands for discrete-time; $z_{k}=e^{j 2 \pi k t / M}$ is the Z-transform variable, and $X(k)$, $E(k)$ and $Q(k)$ the DFTs of $x(t), e(t)$ and $q(t)$, respectively.

One also points out that, in practice, only a limited set of DOFs in $p(t)$ and $q(t)$ are excited and observed, respectively. The identification problem is therefore preferably stated in terms of the measured applied forces $u(t) \in \mathbb{R}^{m \leq r}$ and displacements $y(t) \in \mathbb{R}^{l \leq r}$. Accordingly, the extended input vector is $e(t) \in \mathbb{R}^{\sigma=s+m}$. Equations (7) become

$\left\{\begin{array}{c}z_{k} X(k)=A_{d} X(k)+B_{d}^{e} E(k) \\ Y(k)=C_{d} X(k)+D_{d}^{e} E(k)\end{array}\right.$

where $Y(k)$ is the DFT of $y(t)$ and where the matrices $A_{d}, B_{d}^{e}, C_{d}$ and $D_{d}^{e}$ are now a projection of the original matrices onto the observed and controlled DOFs. The subscript $d$ will be skipped afterwards, because no ambiguity is possible. 


\subsection{Output-state-input matrix equation formulation}

Subspace identification methods are built upon the reformulation of the state-space relations (8) in a matrix form. For this purpose, the measured output frequency spectra matrix is defined as

$Y_{i}=\left(\begin{array}{cccc}Y(1) & Y(2) & \ldots & Y(N) \\ z_{1} Y(1) & z_{2} Y(2) & \ldots & z_{N} Y(N) \\ : & : & : & : \\ z_{1}^{i-1} Y(1) & z_{2}^{i-1} Y(2) & \ldots & z_{N}^{i-1} Y(N)\end{array}\right) \in \mathbb{R}^{l i \times N}$

where $i$ is an user-defined index and $N$ the number of non-necessarily equidistant frequency lines exploited in the identification. Defining $\zeta=\operatorname{diag}\left(z_{1} z_{2} \ldots z_{N}\right) \in \mathbb{R}^{N \times N}, Y_{i}$ is recast into

$$
Y_{i}=\left(\begin{array}{lllll}
Y^{T} & Y^{T} \zeta & Y^{T} \zeta^{2} & \ldots & Y^{T} \zeta^{i-1}
\end{array}\right)^{T} .
$$

The extended input frequency spectra matrix is similarly formed as

$E_{i}=\left(\begin{array}{lllll}E^{T} & E^{T} \zeta & E^{T} \zeta^{2} & \ldots & E^{T} \zeta^{i-1}\end{array}\right)^{T} \in \mathbb{R}^{\sigma i \times N}$.

Introducing the extended observability matrix

$\Gamma_{i}=\left(C^{T}(C A)^{T}\left(C A^{2}\right)^{T} \quad \ldots \quad\left(C A^{i-1}\right)^{T}\right)^{T} \in \mathbb{R}^{l i \times n}$

and the lower-block triangular Toeplitz matrix $H_{i}$

$H_{i}=\left(\begin{array}{ccccc}D^{e} & 0 & 0 & \ldots & 0 \\ C B^{e} & D^{e} & 0 & \ldots & 0 \\ C A B^{e} & C B^{e} & D^{e} & \ldots & 0 \\ : & : & : & : & : \\ C A^{i-2} B^{e} & C A^{i-3} B^{e} & C A^{i-4} B^{e} & \ldots & D^{e}\end{array}\right) \in \mathbb{R}^{l i \times \sigma i}$,

recursive substitution of Equations (8) results in the output-state-input matrix equation

$Y_{i}=\Gamma_{i} X+H_{i} E_{i}$

where $X \in \mathbb{R}^{n \times N}$ is the state spectrum and where the index $i$ is explicitely written to signal the number of block rows of a matrix.

Remark that $i$ and $N$ must be chosen to encompass sufficient valuable information to identify the system. The choice of $N$ is briefly discussed in Section 4 where the possibility to focus on frequency regions of interest will prove to be a major advantage of the FNSI method. A physics-based or information-based decision about $i$ is more delicate. Basically, the larger $i$, the more accurate the identification since $i$ conveys how system dynamics is included in the data matrices. However, redundant information can affect the conditioning of those matrices, hence imposing bounds to $i$. There also exists an obvious trade-off between the values of $i$ and $N$ and the time needed to inverse the model.

\subsection{Estimation of the system matrices}

The FNSI-based computation of the system matrices is a three-step procedure starting from Equation (14). First, an estimate of the extended observability matrix $\Gamma_{i}$ is computed. To this end, the term depending on the input and the nonlinearities in Equation (14), namely $H_{i} E_{i}$, is eliminated using a geometrical projection. Specifically, an orthogonal projection onto the orthogonal complement of $E_{i}$ cancels the extended input term. 
Matrix $\Gamma_{i}$ can then be obtained through a truncated singular value decomposition of the result of the projection. The truncation limits the singular value spectrum to genuine elements, thus removing spurious values and, in turn, reducing the influence of noise and rounding errors on the identification. In addition, the number of retained singular values yields the system order $n$. From the knowledge of $\Gamma_{i}$ and $n$, the second step consists in computing the matrices $A$ and $C$ by means of the shift property $\underline{\Gamma_{i}} A=\overline{\Gamma_{i}}$, where $\Gamma_{i}$ and $\overline{\Gamma_{i}}$ are the matrix $\Gamma_{i}$ without the last and first $l$ rows, respectively. State matrix $\bar{A}$ is consequently found as the least-squares solution of the over-determined system of equations $A=\Gamma_{i}{ }^{\dagger} \overline{\Gamma_{i}}$, where $\dagger$ is the pseudo-inverse, while output matrix $C$ is extracted from $\Gamma_{\mathrm{i}}$ as its first block row. The final step is the estimation of the two system matrices $B^{e}$ and $D^{e}$. A robust resolution scheme that exploits the formulation of a set of linear equations in $B^{e}$ and $D^{e}$, explicitly solved in a least-squares sense, is proposed in reference [7]. For the sake of conciseness, it is not detailed herein.

\subsection{Conversion from state space to physical space}

Once the state-space model $\left(A, B^{e}, C, D^{e}\right)$ is identified, the final step is the estimation of the nonlinear coefficients $\mu_{j}$ and of the FRF matrix of the underlying linear system $H(\omega)$

$H(\omega)=\left(-\omega^{2} M+j \omega C+K\right)^{-1}$.

To achieve this transformation back to physical space, one notes that, in the frequency domain, the substitution of Equation (2) into Equation (1) gives

$H^{-1}(\omega) Q(\omega)+\sum_{j=1}^{s} \mu_{j} b_{j} G_{j}(\omega)=P(\omega)$

where $Q(\omega), G_{j}(\omega)$ and $P(\omega)$ are the continuous-time Fourier transforms of $q(t), g_{j}(t)$ and $p(t)$, respectively. The concatenation of $G_{j}(\omega)$ and $P(\omega)$ then introduces the extended input spectra $E(\omega)$ so as to obtain the linear frequency-domain relationship

$Q(\omega)=H(\omega)\left[\begin{array}{llll}I^{r \times 1} & -\mu_{1} b_{1} & \ldots & -\mu_{s} b_{s}\end{array}\right] E(\omega)=H^{e}(\omega) E(\omega)$

where the matrix $H^{e}(\omega)$ extends the concept of FRF to nonlinear mechanical systems, and encompasses both the underlying linear FRF matrix and the nonlinear coefficients. Moreover, in reference [6], the authors proved that the extended $F R F$ matrix $H^{e}(\omega)$ is an invariant system property and that it can be retrieved, similarly to linear theory, from the combination of the continuous-time state-space matrices

$H^{e}(\omega)=C_{c}\left(j \omega I^{n \times n}-A_{c}\right)^{-1} B_{c}^{e}+D_{c}^{e}$.

As a result, the nonlinear coefficients identified from $H^{e}(\omega)$ using Equations (17) and (18) are spectral quantities, i.e. they are complex-valued and frequency-dependent. A reliable identification scheme together with an appropriate selection of the nonlinear functional forms should make the imaginary parts much smaller than the corresponding real parts. The frequency dependence of the coefficients should also remain small. These indications will serve as quality criteria in Section 4.

\subsection{Identification error criterion}

Because system identification is encapsulated into the more general framework of model validation, which ensures that a model is adequate for its intended use, quantifying the goodness of fit between the experimental data and the system matrices $A, B^{e}, C$ and $D^{e}$ generated from these data is fundamental. In this section, a robust error criterion utilising the four system matrices is defined. 
Considering again the output-state-input Equation (14) and pre-multiplying by the orthogonal complement of $\Gamma_{\mathrm{i}}$, one obtains

$\Gamma_{\mathrm{i}}^{\perp}\left(Y_{i}-H_{i} E_{i}\right)=0$.

This expression can be used to quantify how good the estimated state-space matrices gathered in $H_{i}$ fit the I-O data $\left(Y_{i}\right.$ and $\left.E_{i}\right)$. Specifically, the proposed indicator is the deviation of the largest principal angle between the subspaces spanned by the rows of $\Gamma_{\mathrm{i}}^{\perp}$ and the columns of $Y_{i}-H_{i} E_{i}$, termed $\theta$ in the paper, from its ideal value of 90 degrees. As shown in Section 4, this error criterion will prove particularly useful for estimating the clearances beyond which the mechanical stops of the SmallSat spacecraft are reached. This adds some characterisation capabilities to the FNSI method.

\section{THE SMALLSAT SPACECRAFT STRUCTURE}

The SmallSat structure was conceived by EADS-Astrium as a low-cost platform for small satellites in low earth orbits. It is a monocoque tube structure which is $1.2 \mathrm{~m}$ long and $1 \mathrm{~m}$ large. It incorporates eight flat faces for equipment mounting purposes, creating an octagon shape, as shown in Figure 1. The octagon is manufactured using carbon fibre reinforced plastic by means of a filament winding process. The structure thickness is $4 \mathrm{~mm}$ with an additional $0.25 \mathrm{~mm}$ thick skin of Kevlar applied to both the inside and outside surfaces to provide protection against debris. The interface between the spacecraft and launch vehicle is achieved through four aluminium brackets located around cut-outs at the base of the structure. The total mass including the interface brackets is around $64 \mathrm{~kg}$.

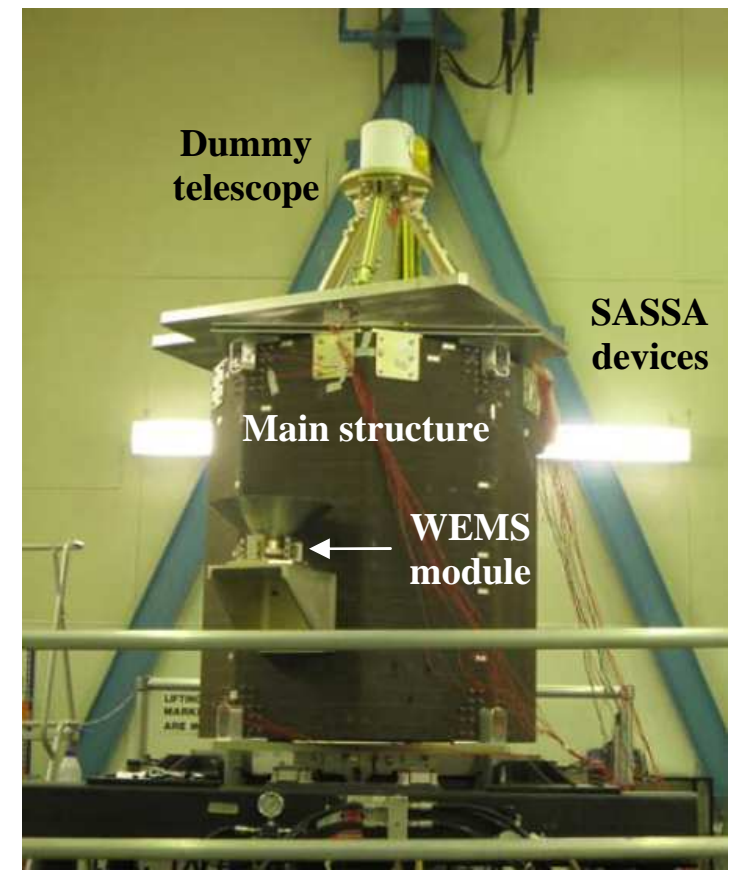

Figure 1: SmallSat spacecraft with the WEMS module and the dummy telescope connected to the main structure via the SASSA devices.

The spacecraft structure supports a dummy telescope composed of two stages of base-plates and struts supporting various concentrated masses; its mass is around $140 \mathrm{~kg}$. The dummy telescope plate is connected to the SmallSat top floor via three shock attenuators, termed SASSAs (Shock Attenuation System for Spacecraft and Adaptor), the behaviour of which is considered as linear in the present study. The top floor is an $1 \mathrm{~m}^{2}$ sandwich aluminium panel, with $25 \mathrm{~mm}$ core and $1 \mathrm{~mm}$ skins. Besides, as depicted in Figure 2 (a), a support bracket connects to one of the eight walls the so-called Wheel Elastomer Mounting System (WEMS) device which is loaded with an $8 \mathrm{~kg}$ dummy inertia wheel. The 
purpose of this device is to isolate the spacecraft and the payload from disturbances coming from the inertia wheel through the presence of a soft elastomeric interface between its mobile part, i.e. the inertia wheel and a supporting metallic cross, and its fixed part, i.e. the bracket and by extension the spacecraft. Moreover, mechanical stops limit the axial and lateral motions of the WEMS mobile part during launch, which gives rise to strongly nonlinear dynamical phenomena. Figure 2 (b) presents a simplified though relevant modelling of the WEMS device where the inertia wheel is seen as a point mass. The four nonlinear connections (NCs) between the WEMS mobile and fixed parts are labelled NC $1-4$. Each NC possesses a trilinear spring in the axial direction (elastomer in traction plus two stops), a bilinear spring in the radial direction (elastomer in shear plus one stop) and a linear spring in the third direction (elastomer in shear). In Figure 2 (b), linear and nonlinear springs are denoted by squares and circles, respectively.
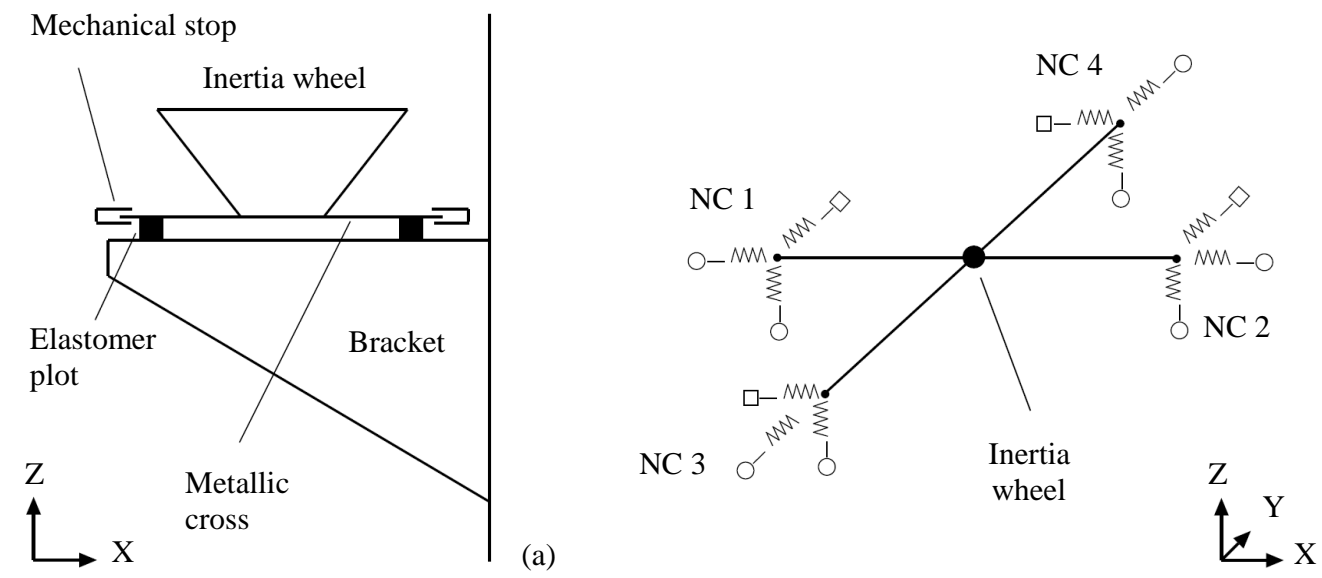

Figure 2: WEMS device. (a) Detailed description of the WEMS components; (b) simplified modelling of the WEMS mobile part considering the inertia wheel as a point mass. The linear and nonlinear connections between the WEMS mobile and fixed parts are signalled through squares and circles, respectively.

A finite element model of the SmallSat was developed and is used in the present work to conduct numerical experiments. It comprises about 65,000 DOFs and the comparison with experimental data revealed its good predictive capabilities. The model consists of shell elements (main structure, topfloor panels and WEMS) and point masses (inertia wheel and telescope) and meets boundary conditions by means of four clamped nodes. Structural damping is considered via the proportional damping assumption and lumped dashpots are utilised to model dissipation in the elastomer plots, hence resulting in a highly non-proportional damping matrix. Finally, nonlinearity is introduced within the WEMS module by means of eight piecewise-linear springs whose parameters are given in Table 1 through adimensional values for confidentiality reasons.

\begin{tabular}{|c|c|c|c|}
\hline & Clearance & Linear slope & Nonlinear slope \\
\hline Axial Z & 1 & 7.58 & 100 \\
\hline Lateral X and Y & 2 & 1.97 & 39.7 \\
\hline
\end{tabular}

Table 1: Adimensional parameters of the WEMS stiffness curves.

Numerical simulations were carried out using a nonlinear Newmark integration scheme, considering a sampling frequency of 2,500 Hz. A periodic noise forcing with root-mean-squared (RMS) value of 200 $N$ was applied to the inertia wheel in the symmetric $\mathrm{X}-\mathrm{Y}$ direction. It consisted of a single bandlimited $(5-50 \mathrm{~Hz})$ normally-distributed random signal (30,000 samples, 12 seconds) repeated 8 times. This excitation caused the strong and comparable activation of all WEMS nonlinearities, as confirmed in Table 2 where the number of clearance exceedances in the eight nonlinear springs is reported. Filtered white noise in $0-100 \mathrm{~Hz}$ was finally added to the synthetic time series to recreate the 
mechanical and electrical disturbances observed in a typical measurement setup. The noise level was selected as $0.5 \%$ of the RMS response amplitude at the bracket tip.

\begin{tabular}{|c|c|c|c|c|}
\hline & NC 1 & NC 2 & NC 3 & NC 4 \\
\hline $\mathrm{X}$ & 14,803 & 13,600 & - & - \\
\hline $\mathrm{Y}$ & - & - & 13,453 & 13,001 \\
\hline $\mathrm{Z}$ & 31,661 & 25,854 & 28,043 & 21,218 \\
\hline
\end{tabular}

Table 2: Number of clearance exceedances. No entry means the absence of mechanical stop.

\section{SMALLSAT IDENTIFICATION BASED ON NOISY DATA}

The first step in formulating a nonlinear subspace model is the selection of an adequate order. This step is crucial since too low orders involve unmodeled dynamics whereas too large orders increase the noise sensitivity of the model. In linear subspace applications, the decision is most frequently based upon the analysis of a stabilisation diagram [3]. An appealing asset of the FNSI method is that it still supports the use of this decision-making tool in the presence of nonlinearity in I-O data, because it succeeds in decoupling linear parameters from nonlinear distortions. Figure 3 depicts the stabilisation of the linear natural frequencies, damping ratios and complex modes shapes for model orders up to 60 and indicates that the order 10 is appropriate. Note that stabilisation is verified between successive orders, e.g. $n$ and $n+2$, taking as reference the linear properties calculated at order $n$. This explains why the order 10 in Figure 3 is seemingly unstabilised though the opposite results from an inspection at order 12.

Besides the model order, the adequate selection of the functional forms $g_{j}(t)$ also heavily determines the quality of the identified model. In the case of the WEMS device, the use of piecewise-linear functions is obvious but the selection of the associated clearances remains a challenging task. The error criterion introduced in Section 2.6 can help make a decision by selecting the model parameters which give the angle $\theta$ closest to 90 degrees. In practice, we seek the minimum of the normalised indicator $1-2 * \theta / \pi$ which varies between 0 and 1 . Figure 4 presents this indicator in logarithmic scaling for different axial and lateral clearances. It is found to retrieve the exact values as a clear minimum is observed for $(1,2)$. Note that, rather than visually inspecting the error criterion, the estimation of the clearances could also be achieved using optimisation routines, but the presence of local minima in the objective function would require a gradient-free scheme, e.g. a genetic or simplexbased algorithm.

The frequency variation of the eight identified nonlinear coefficients is presented in Figure 5, considering the $5-40 \mathrm{~Hz}$ band since the modes governing the system response are located around 10 and $30 \mathrm{~Hz}$. The corresponding numerical values are listed in Table 3 . The real parts of the lateral and axial coefficients remain bounded within 10 and $20 \%$-intervals around their exact values, respectively, and the imaginary parts are generally more than two orders of magnitude smaller. Despite the high dimensionality of the identification problem ( 1 input, 18 outputs, 8 nonlinearities, 180,000 time samples and 75 block rows), the inversion of the model was obtained in 105 seconds thanks to restriction of the frequency samples to the input band, hence encompassing only 3,240 frequency lines.

The relative errors on the estimated natural frequencies and damping ratios of the underlying linear spacecraft are given in Table 4, together with the modal assurance criterion (MAC) between the identified and exact complex mode shapes. The linear properties of the structure are almost perfectly recovered from strongly nonlinear measurements. All these results demonstrate the excellent identification capabilities of the FNSI method. This is also confirmed in Figure 6 where FNSI accurately reconstructs the linear driving-point FRF while the H1 estimate is found to suffer from important nonlinear distortions. 


\begin{tabular}{|c|c|c|c|}
\hline Nonlinear coefficient & Averaged value & Error $(\%)$ & Ratio real/imag. (log) \\
\hline NC $1-\mathrm{X}$ & 39.77 & 0.18 & 3.17 \\
\hline NC $2-\mathrm{X}$ & 39.70 & 0.00 & 2.81 \\
\hline NC $3-\mathrm{Y}$ & 39.80 & 0.27 & 3.93 \\
\hline NC $4-\mathrm{Y}$ & 39.93 & 0.59 & 3.08 \\
\hline NC $1-\mathrm{Z}$ & 100.65 & 0.65 & 2.63 \\
\hline NC $2-\mathrm{Z}$ & 100.17 & 0.17 & 2.24 \\
\hline NC $3-\mathrm{Z}$ & 99.99 & -0.01 & 2.33 \\
\hline NC $4-\mathrm{Z}$ & 100.17 & 0.17 & 3.25 \\
\hline
\end{tabular}

Table 3: Estimated nonlinear stiffness coefficients averaged over $5-40 \mathrm{~Hz}$ (real parts), relative errors and ratios (in logarithmic scale) between real and imaginary parts.

\begin{tabular}{|c|c|c|c|c|c|}
\hline Mode & $\begin{array}{c}\text { Id. natural } \\
\text { frequency }(\mathrm{Hz})\end{array}$ & Error $(\%)$ & $\begin{array}{c}\text { Id. damping } \\
\text { ratio (\%) }\end{array}$ & Error (\%) & Complex MAC \\
\hline 1 & 10.69 & 0.20 & 5.33 & -0.16 & 1.00 \\
\hline 2 & 11.03 & 0.21 & 5.59 & 0.13 & 1.00 \\
\hline 3 & 28.23 & -0.12 & 9.51 & -2.86 & 1.00 \\
\hline 4 & 28.41 & 0.02 & 10.41 & -1.92 & 0.96 \\
\hline 5 & 30.12 & 0.04 & 6.98 & -0.78 & 1.00 \\
\hline
\end{tabular}

Table 4: Identified natural frequencies and damping ratios and relative errors with the exact underlying linear properties; MAC values between identified and exact complex mode shapes.

\section{CONCLUSIONS}

This paper aimed at identifying the SmallSat spacecraft, a strongly nonlinear space structure developed by EADS-Astrium, from noisy synthetic data. For this purpose, a nonlinear generalisation of subspace methods formulated in the frequency domain, referred to as the FNSI method, was exploited. The amplitudes of the nonlinear internal forces and the underlying linear parameters of the structure were shown to be accurately and rapidly estimated by this approach. The problem of selecting the model which best fits input-output data was also tackled through the use of a modelling error criterion. This study is arguably one of the first successful identifications of such a complex reallife nonlinear structure in the technical literature.

However, additional investigations are needed to further assess the capabilities of the FNSI method. In particular, the variability of the identification over repeated experiments due to the random nature of the excitation and the noise should be carefully analysed. A particular attention should also be devoted to the asymptotic behaviour of the proposed subspace algorithm for an increasing number of measured samples and block rows. Finally, the use of stabilisation diagrams in nonlinear system identification, initiated in the present paper, should also be studied in more detail.

\section{ACKNOWLEDGEMENTS}

This paper has been prepared in the framework of the European Space Agency (ESA) Technology Research Programme study "Advancement of Mechanical Verification Methods for Non-linear Spacecraft Structures (NOLISS)" (ESA contract No.21359/08/NL/SFe - Grant manager: A. Newerla). The author J.P. Noël would also like to acknowledge the Belgian National Fund for Scientific Research (FRIA fellowship) for its financial support. 


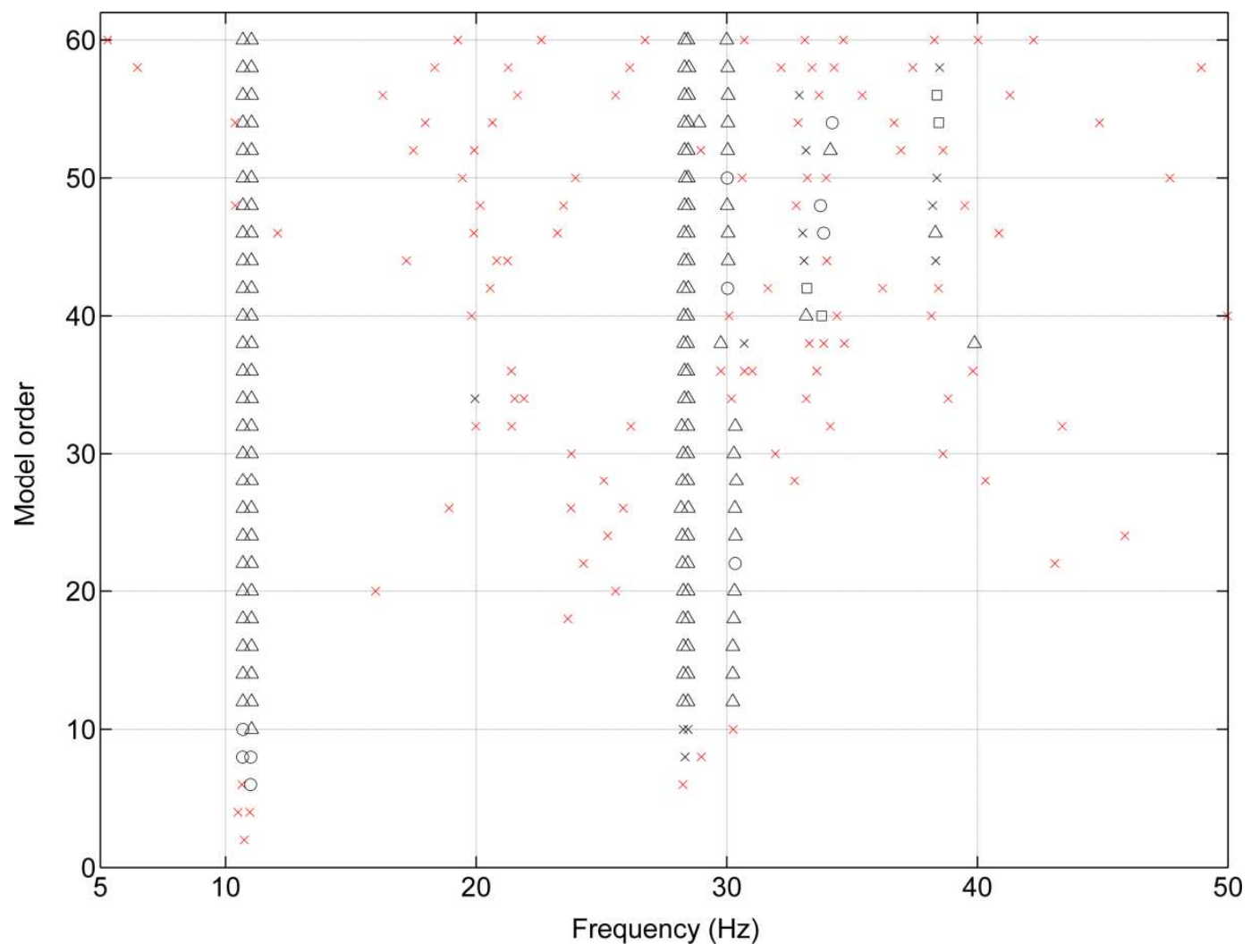

Figure 3: Stabilisation diagram. Red cross: new pole; black cross: stabilisation in natural frequency; square: extra stabilisation in damping ratio; circle: extra stabilisation in complex mode shape; triangle: full stabilisation. Stabilisation thresholds in natural frequency, damping ratio and complex modal assurance criterion (MAC) value are $0.5 \%, 2 \%$ and 0.98 , respectively.

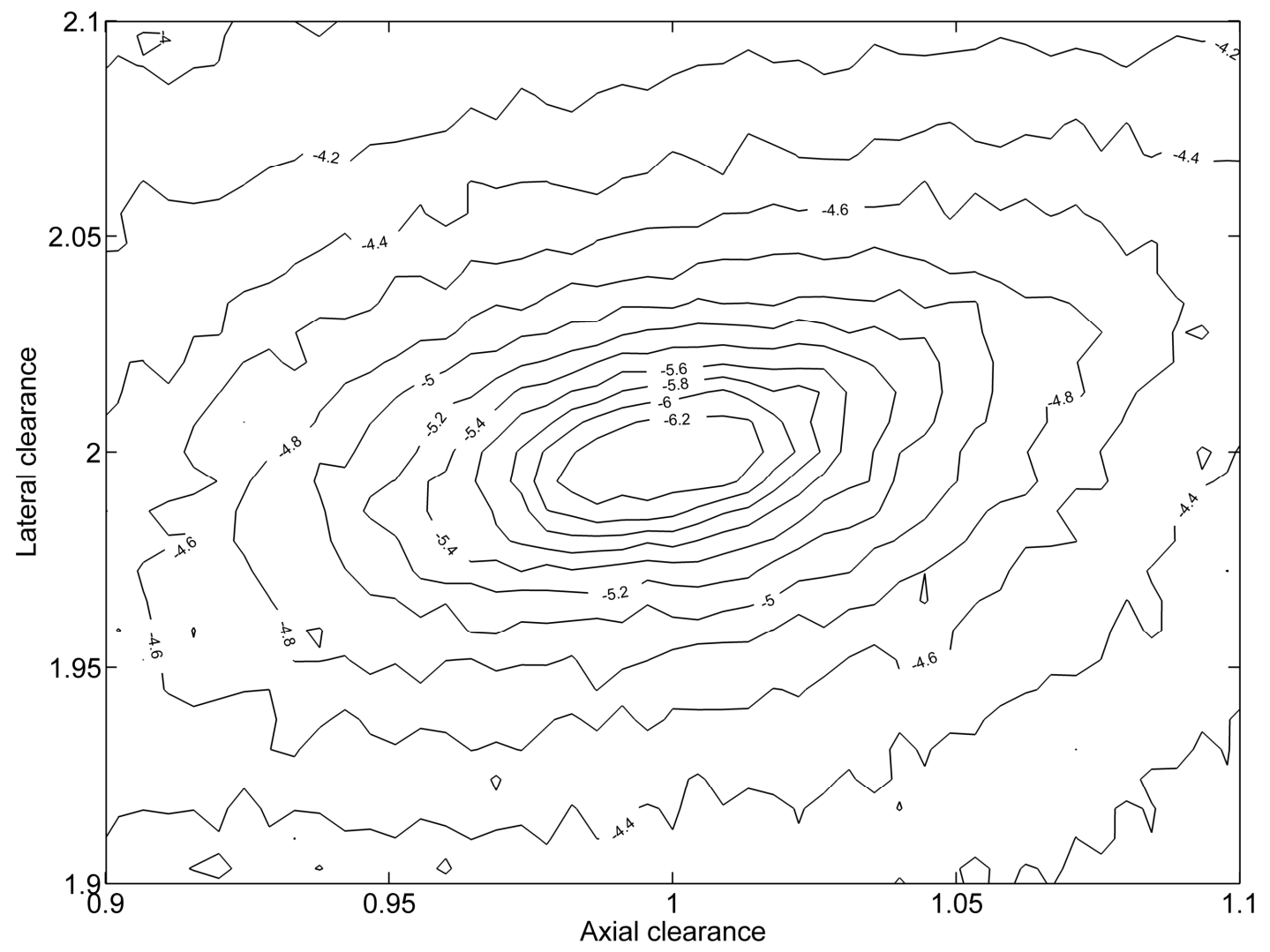

Figure 4: Estimation of the axial and lateral clearances using a contour plot of the normalised error criterion in logarithmic scaling. 

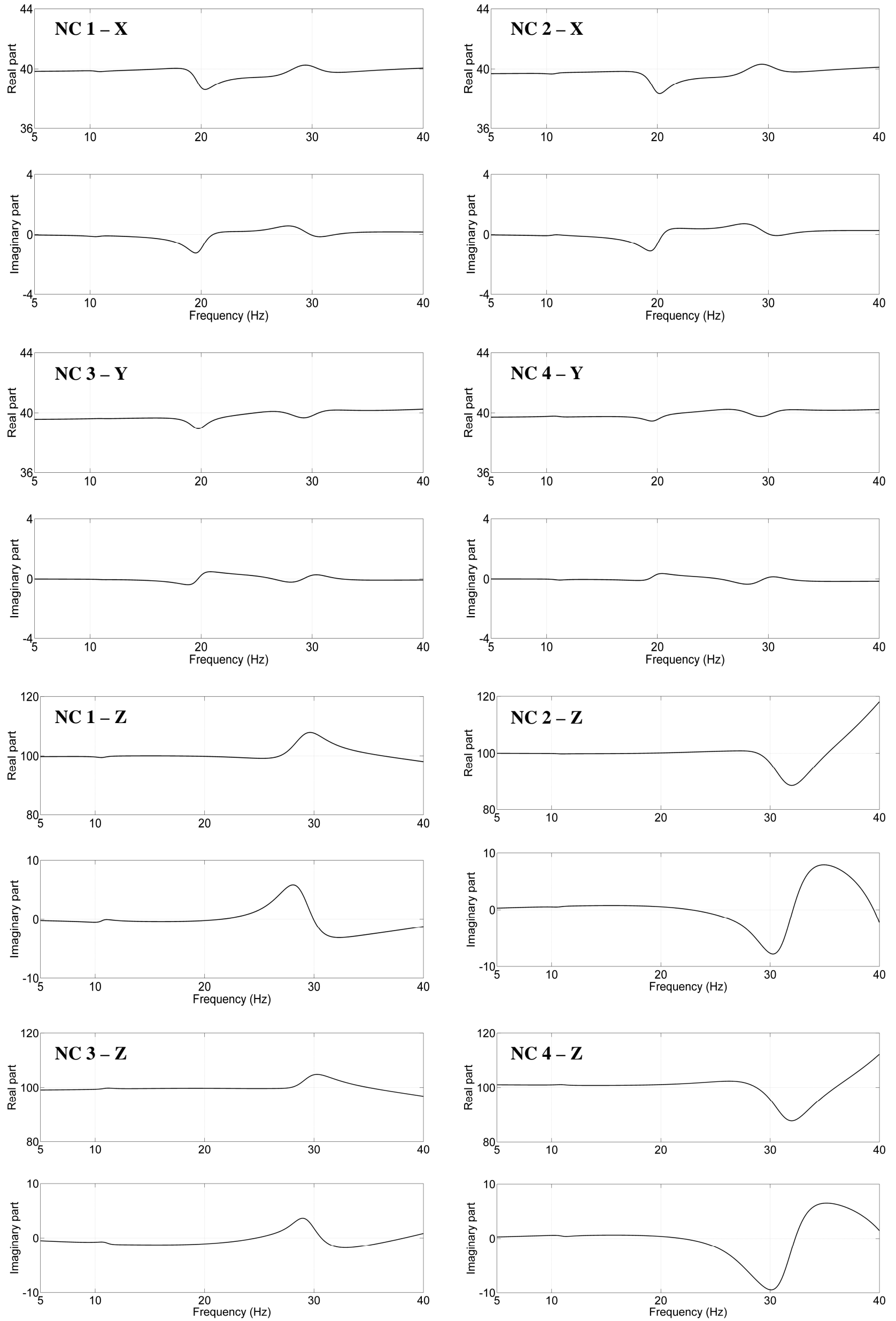

Figure 5: FNSI estimation of the eight nonlinear stiffness coefficients. The real parts of the lateral and axial coefficients are displayed within 10 and $20 \%$-error bounds, respectively. 


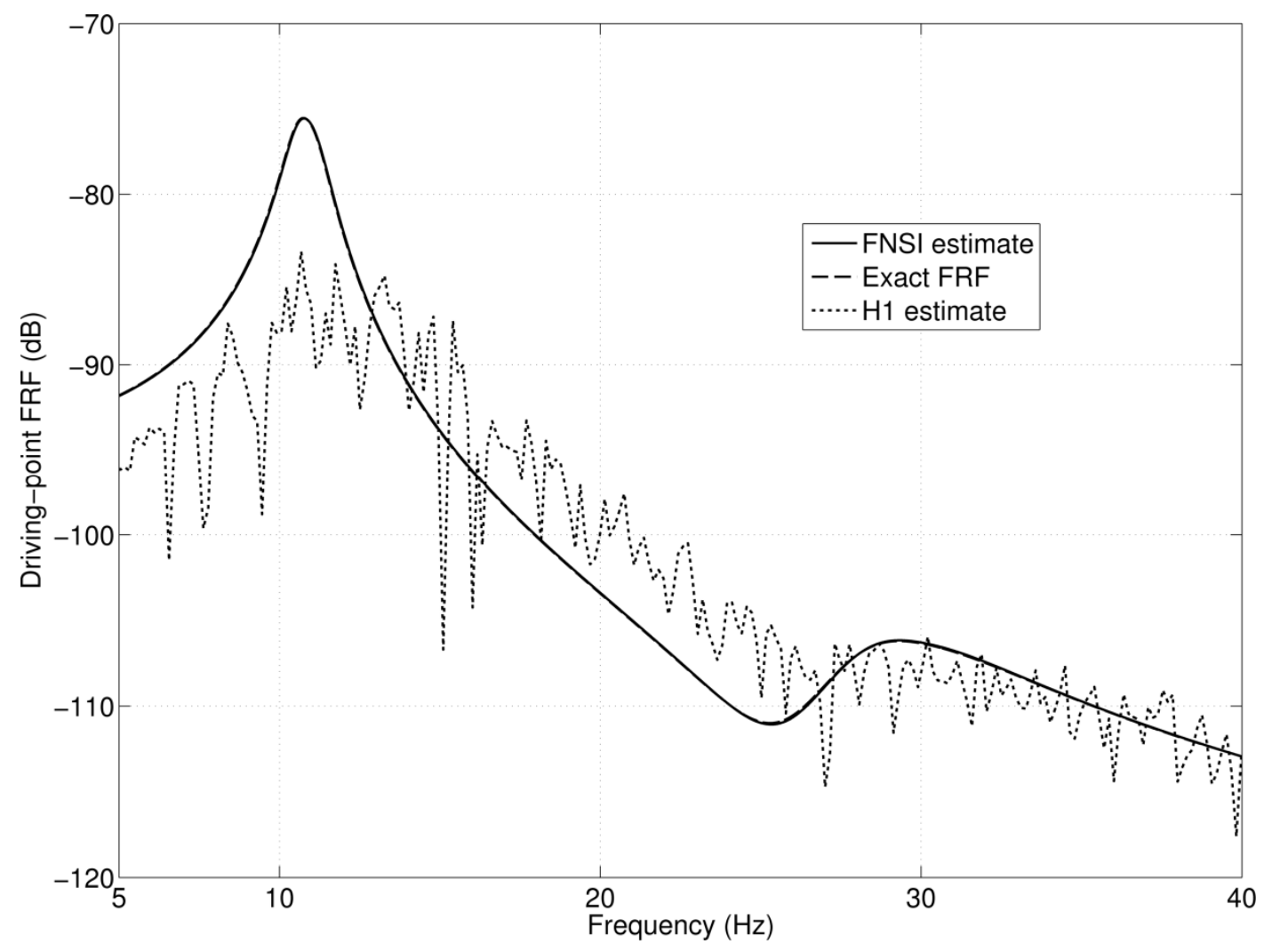

Figure 6: Reconstruction of the underlying linear driving-point FRF from nonlinear data and comparison with the exact FRF and the H1 linear estimate.

\section{REFERENCES}

[1] P. Van Overschee, B. De Moor (1996) Subspace Identification for Linear Systems. Boston/London/Dordrecht, Kluwer Academic Publishers

[2] B. Peeters, H. Van Der Auweraer, P. Guillaume (2004) The PolyMAX frequency-domain method: a new standard for modal parameter estimation. Shock and Vibration 11: 395-409

[3] E. Reynders, G. De Roeck (2008) Reference-based combined deterministic-stochastic subspace identification for experimental and operational modal analysis. Mechanical Systems and Signal Processing 22: 617-637

[4] L. Mevel, L. Hermans, H. Van Der Auweraer (1999) Application of subspace-based fault detection method to industrial structures. Mechanical Systems and Signal Processing 13: 823-838

[5] S. L. Lacy, D. S. Bernstein (2005) Subspace identification for non-linear systems with measuredinput non-linearities. International Journal of Control 78: 906-926

[6] S. Marchesiello, L. Garibaldi (2008) A time domain approach for identifying nonlinear vibrating structures by subspace methods. Mechanical Systems and Signal Processing 22: 81-101

[7] J.P. Noël, G. Kerschen (2012) A new subspace-based approach to identify nonlinear mechanical structures in the frequency domain. In: Proc. of the 16th IFAC Symposium on System Identification, Brussels, Belgium

[8] G. Kerschen, K. Worden, A. F. Vakakis, J.C. Golinval (2006) Past, present and future of nonlinear system identification in structural dynamics. Mechanical Systems and Signal Processing 20: 505-592

[9] D. E. Adams, R. J. Allemang (2000) A frequency domain method for estimating the parameters of a non-linear structural dynamic model through feedback. Mechanical Systems and Signal Processing 14(4): 637-656

[10] R. Pintelon, J. Schoukens (2001) System Identification: A Frequency Domain Approach. Piscataway, IEEE Press

[11] P. Van Overschee, B. De Moor (1996) Continuous-time frequency domain subspace system identification. Signal Processing 52: 179-194 